\title{
Moving cardiology to the front of the hospital
}

The major factor influencing the outcome of thrombolytic therapy for acute myocardial infarction is the "door to needle" time. For every hour that thrombolytic treatment is delayed after the onset of symptoms, 1.6 lives are lost per 1000 patients treated. ${ }^{1}$ In many institutions the door to needle time exceeds one hour.

Various attempts have been made to reduce unacceptable treatment delays. The most successful approach has been to shift the administration of thrombolytic treatment from the coronary care unit (CCU) to the emergency department. ${ }^{2}$ In the study by Hourigan and colleagues which appeared in a recent issue of Heart, ${ }^{3}$ the door to needle times of 100 patients treated by emergency physicians in the emergency department were prospectively compared with those of a historical group of 89 non-consecutive patients treated in the CCU between three years and 16 months previously. Not surprisingly, the door to needle times in the emergency department were much shorter than those in the CCU (37 $v 80$ minutes). This is an admirable achievement, and patient outcomes must have been improved as a result. However, according to current guidelines, the door to needle delay should have been no longer than 30 minutes, ${ }^{45}$ and the usage of thrombolytic treatment, which was given to only $20 \%$ of patients with a subsequent discharge diagnosis of myocardial infarction, was rather low. ${ }^{6}$

To evaluate the appropriateness of the treatment given in the emergency department and the CCU, one would need to know how well the ECG criteria and contraindications against thrombolytic treatment were adhered to. Judging by their discharge diagnoses, thrombolytic treatment was inappropriately given to one patient in the CCU and three in the emergency department who did not have myocardial infarction. There was a significantly higher mortality rate among the patients who received treatment in the emergency department $(12 \% \quad v 3.4 \%$ in the CCU, $\mathrm{p}=0.02$ ), including two deaths that were attributed to errors of judgement.

In this selected population, it is possible that some patients receiving thrombolytic treatment in the CCU initially had non-diagnostic ECGs but subsequently fulfilled the ECG criteria for thrombolytic treatment, thus biasing the analysis against this group. A temporal ascertainment bias could also have occurred-that is, sicker patients may have died before reaching the CCU, affecting both the door to needle times and clinical outcome measurements. The patients given thrombolytic treatment in the CCU may also have been older or could have had more complex histories, hypertension requiring initial treatment, or left bundle branch block - all of which could have delayed treatment. Delays could also have occurred while staff retrieved patient records to establish whether conduction abnormalities were new or old. In these instances, patients should be deemed to have new onset bundle branch block and receive treatment promptly. If there is any doubt that the pain is ischaemic in nature, myoglobin or troponin tests should be performed at the bedside. ${ }^{4}$ Echocardiography may also be helpful.

\section{Other ways to reduce door to needle times}

There are other ways to reduce door to needle times besides administering thrombolytic treatment in the emergency department-for example, direct admission to the CCU if myocardial infarction has been diagnosed by the general practitioner, ${ }^{7}$ dedicated chest pain units, ${ }^{8}$ fast tracking policies within hospitals, ${ }^{9}$ and pre-hospital administration of thrombolytic treatment. ${ }^{10}$ Alternatively, patients presenting to the emergency department with ischaemic chest pain could be given an ECG within 10 minutes and be moved promptly to the CCU if the ECG fulfils the criteria for thrombolytic eligibility. ${ }^{4}$

There are two causes of delay when thrombolytic treatment is administered in the CCU. The first is the time it takes to transfer the patient from the emergency department. In many hospitals, the CCU is a long way from the emergency department, and so it may well be more practical to administer thrombolytic treatment in the emergency department.

The second delay occurs between the arrival of the patient in the CCU and assessment by a CCU physician. This can be minimised by reading the ECG immediately on arrival and not repeating the history already taken in the emergency department, other than checking for contraindications against thrombolytic treatment (particularly aortic dissection) by appropriate questioning and examination. It is vital that the administration of thrombolytic treatment is not delayed for lengthy bedside consultations or the ordering of further tests such as chest $x$ rays.

When thrombolytic treatment is initiated in the emergency department, the patient should be transferred immediately to the CCU rather than being detained for monitoring until the infusion is completed, as sometimes occurs. The use of bolus thrombolytic agents such as reteplase and tenecteplase can go a long way towards minimising the time patients spend in the emergency department. ${ }^{11} 12$

\section{Chest pain units}

An important change in practice in recent years has been the development of chest pain units. ${ }^{13}$ The purpose of a chest pain unit is not just for triage, but includes administration of thrombolytic treatment, ruling out myocardial infarction where applicable, detecting myocardial ischaemia, and discharging low risk patients after 6-12 hours. There should be treadmill and echocardiographic facilities readily available in these units, and educational materials and advice about risk factor modification should be provided. Appropriate follow up should be arranged for 
patients who are discharged. Low risk patients can be identified for early discharge by same day stress testing for ischaemia (using exercise treadmill, stress echocardiography or nuclear stress testing), the use of appropriate myocardial markers, monitoring for ischaemia and arrhythmias, and repeat risk profiling before discharge. In the USA, approximately $22 \%$ of emergency departments have a chest pain unit. A number of studies have compared the outcomes achieved by chest pain units with routine practice. ${ }^{813}$ There were no differences in mortality or missed diagnoses, but then these were only small studies. Some studies have reported that chest pain units actually reduced treatment costs. ${ }^{8}$

Although the difference was not significant, it is concerning to note that in the study by Hourigan and colleagues, only $8 \%$ of patients receiving thrombolytic treatment in the emergency department were randomised into clinical trials, compared with $11.2 \%$ of patients receiving treatment in the CCU. ${ }^{3}$ This may have been because of the number of trials being performed at the time of review. It is vital, however, that research aimed at improving reperfusion strategies and patient outcomes is not stymied by audit of other aspects of patient care. Participation in clinical trials is a very effective way to improve clinical practice, and trial enrolment should be counted as an outcome measure.

The shifting of thrombolytic administration from the CCU to the emergency department does raise concerns about the impact on the quality of patient care and the training of cardiologists and CCU nurses. While time is of the essence when administering thrombolytic treatment, there are other components of patient care that are best handled by dedicated CCU staff. The diagnosis and choice of management strategy are complex issues in patients with acute coronary syndromes, and require considerable training, expertise, and non-competitive clinical time. CCU nurses are experienced in monitoring for arrhythmias and ischaemia, recognising complications quickly (for example, cardiac rupture), and giving appropriate reassurance and information to patients and relatives about proposed treatments, prognoses, and rehabilitation. Their professional development and career satisfaction might suffer if their involvement in the initial treatment of acute coronary syndromes were to be curtailed. The education of cardiology registrars, too, requires training at the bedside to gain experience in the recognition and management of acute ischaemia.

\section{New treatment strategies}

New treatment strategies for patients with ST elevation acute coronary syndromes may include administration of a bolus thrombolytic agent, a glycoprotein IIb/IIIa inhibitor, a direct antithrombin or low molecular weight heparin, ST segment monitoring for evidence of reperfusion failure, acute angiography in some or all patients, and facilitated percutaneous revascularisation. The treatment of patients with non-ST elevation acute coronary syndromes may include a IIb/IIIa inhibitor, low molecular weight heparin, perhaps a direct antithrombin, intra-aortic balloon pumping, and percutaneous revascularisation.
The triage of patients to an appropriate treatment strategy requires a detailed history, clinical examination, risk profiling, expert interpretation of ECGs (including identification of posterior infarction, ischaemia with paced arrhythmias or baseline ECG abnormalities), measurement of troponins and new inflammatory markers and, in some patients, echocardiography, computed tomographic scanning, nuclear scanning, or magnetic resonance imaging. The integration of all of this information in a timely manner presents a considerable challenge.

Late patient presentation remains the greatest barrier to expeditious reperfusion, and constitutes two thirds of the total time from symptom onset to the initiation of reperfusion treatment. ${ }^{14}$ The development of a partnership between emergency physicians and cardiologists is a very positive step. However, although door to needle delays can be greatly reduced by shifting the administration of thrombolytic treatment to the emergency department, patients would be unlikely to benefit from moving the rest of acute cardiology to the front of the hospital. Cardiologists must not give up the emergency aspects of their specialty.

Director of Coronary Care and Cardiovascular Research,

HARVEY D WHITE

Green Lane Hospital,

Auckland, New Zealand,

harveyw@ahsl.co.nz

1 Fibrinolytic Therapy Trialists' (FTT) Collaborative Group. Indications for fibrinolytic therapy in suspected acute myocardial infarction: collaborative overview of early mortality and major morbidity results from all randomised overview of early mortality and major morbidity results from

2 Maynard C, Weaver WD, Lambrew C, et al. Factors influencing the time to administration of thrombolytic therapy with recombinant tissue plasminogen activator (data from the national registry of myocardial infarction). Participants in the national registry of myocardial infarction. Am f Cardiol 1995;76:548-52.

3 Hourigan CT, Mountain D, Rogers IR, et al. Changing the site of delivery of thrombolytic treatment for acute myocardial infarction from the coronary care unit to the emergency department greatly reduces door to needle time. Heart 2000;84:157-63.

4 White HD, Van de Werf FJJ. Clinical cardiology: new frontiers: thrombolysis for acute myocardial infarction. Circulation 1998;97:1632-46.

5 Ryan TJ, Antman EM, Brooks NH, et al. 1999 update: ACC/AHA guidelines for the management of patients with acute myocardial infarction: a report of the American College of Cardiology/American Heart Association task force on practice guidelines (committee on management of acute myocardial infarction). F Am Coll Cardiol 1999;34:890-911.

6 French JK, Williams BF, Hart HH, et al. Prospective evaluation of eligibility for thrombolytic therapy in acute myocardial infarction. BMF 1996;312: 1637-41.

7 Prasad N, Wright A, Hogg KJ, et al. Direct admission to the coronary care unit by the ambulance service for patients with suspected myocardial infarction. Heart 1997;78:462-4.

8 Goodacre SW. Should we establish chest pain observation units in the UK? A systematic review and critical appraisal of the literature. $f$ Accid Emerg

9 Porter G, Doughty R, Gamble G, et al. Thrombolysis in acute myocardial Porter G, Doughty R, Gamble G, et al. Thrombolysis in acute myocardial
infarction: reducing in hospital treatment delay. NZ Med $\mathcal{f} 1995 ; 108: 253-$ infa

10 The GREAT Group. Feasibility, safety, and efficacy of domiciliary thrombolysis by general practitioners: Grampian region early anistreplase trial. $B M \mathcal{F}$ 1992;305:548-53.

11 The Global Use of Strategies to Open Occluded Coronary Arteries (GUSTO III) Investigators. A comparison of reteplase with alteplase for acute myocardial infarction. N Engl f Med 1997;337:1118-23.

12 Assessment of the Safety and Efficacy of a New Thrombolytic (ASSENT-2) Investigators. Single-bolus tenecteplase compared with front-loaded alteplase in acute myocardial infarction: the ASSENT-2 double-blind randomised trial. Lancet 1999;354:716-22.

13 Farkouh ME, Smars PA, Reeder GS, et al. A clinical trial of a chest-pain observation unit for patients with unstable angina. N Engl f Med 1998;339: observation $1882-8$.

14 Weaver WD, Cerqueira M, Hallstrom AP, et al. Prehospital-initiated vs hospital-initiated thrombolytic therapy. $\mathscr{F} A M A$ 1993;270:1211-6. 